\title{
miR-210-3p Promotes Lung Cancer Development and Progression by Modulating USFI and PCGF3
}

\author{
Qian Chen (D) ${ }^{1,2, *}$ \\ Hongyu Zhang (D) ${ }^{3,4, *}$ \\ Jianyin Zhang (D) ${ }^{1,2}$ \\ Le Shen (iD) ${ }^{1,2}$ \\ Jing Yang (D) ${ }^{1,2}$ \\ Yan Wang (D) ${ }^{1,2}$ \\ JinXiu $\mathrm{Ma} \mathbb{D}^{1,2}$ \\ Bing Zhuan iD ${ }^{1,2}$ \\ 'Department of Respiratory Medicine, \\ People's Hospital of Ningxia Hui \\ Autonomous Region, Yinchuan, People's \\ Republic of China; ${ }^{2}$ Department of \\ Respiratory Medicine, First Affiliated \\ Hospital of Northwest Minzu University, \\ Yinchuan, People's Republic of China; \\ ${ }^{3}$ Department of Intervention and \\ Vascular Surgery, People's Hospital of \\ Ningxia Hui Autonomous, Yinchuan, \\ People's Republic of China; ${ }^{4}$ Department \\ of Intervention and Vascular Surgery, \\ First Affiliated Hospital of Northwest \\ Minzu University, Yinchuan, People's \\ Republic of China \\ *These authors contributed equally to \\ this work
}

\begin{abstract}
Purpose: Lung cancer represents one of the most frequent solid tumors. Adenocarcinoma is a common type of tumor and a significant threat to individual health globally. MicroRNAs (miRNAs) are recognized as critical governors of gene expression during carcinogenesis, while their effects on lung cancer occurrence and development are required for further investigation. Herein, the functional role of miR-210-3p and its regulation mechanism were characterized in lung cancer.
\end{abstract}

Methods: A total of 50 pairs of tumor and tumor-free lung tissues were surgically resected from lung cancer patients. Dual-luciferase reporter assay and RNA immunoprecipitation assay were performed to examine USF1 binding with miR-210-3p and PCGF3. Cultured human lung cancer cells A549 were assayed for viability, apoptosis, migration, and invasion in vitro by CCK-8 test, flow cytometry, transwell chamber assays, tumorigenesis, and lymph node metastasis in vivo by mouse xenograft experiments.

Results: miR-210-3p was upregulated in lung cancer tissues. The inhibition of miR-210-3p by specific inhibitor tempered lung cancer development and metastasis in vitro and in vivo. miR-210-3p targeted USF1 and inhibited its expression. USF1 was bound with PCGF3, which increased its transcription. PCGF3-specific knockdown mimicked the effect of miR$210-3 p$ on lung cancer development and metastasis in vitro and in vivo.

Conclusion: The current study demonstrated that miR-210-3p facilitates lung cancer development and metastasis by impairing USF1-mediated promotion of PCGF3, which provides a better understanding of the mechanism of lung cancer development and metastasis.

Keywords: lung cancer, miR-210-3p, USF1, PCGF3

\section{Introduction}

Lung cancer, together with liver and colorectal cancer, remains a leading cause of cancer-related deaths worldwide, and $80 \%$ of lung cancers belong to non-small cell lung cancers (NSCLCs). ${ }^{1}$ Reportedly, numerous risk factors, including tobacco use, occupational carcinogens, exposure to radon, and environmental deterioration, are responsible for lung cancer. ${ }^{2}$ The incidence rate of lung cancer is rising, and the prognosis is poor. ${ }^{3}$ In the recent three decades, the 5-year survival of patients with lung cancer remains only $19 \%$, with minimal improvement. ${ }^{4}$ Thus, it is imperative to explore the underlying molecular mechanisms and identify the novel prognostic biomarkers and potential therapeutic targets for lung cancer.

MicroRNAs (miRNAs) are endogenous small non-coding single-stranded RNAs that regulate the expression of downstream genes by binding with the $3^{\prime}$ untranslated region (3'-UTR) of the target mRNAs. ${ }^{5}$ Accumulating evidence revealed that several miRNAs are involved in the regulation of diverse
Correspondence: Bing Zhuan Department of Respiratory Medicine, First Affiliated Hospital of Northwest Minzu University, No. 30I Zhengyuan North Street, Jinfeng District, Yinchuan, 750004, People's Republic of China Tel +86 I87095I8I79

Email Zhuanb5I8@I63.com 
biological processes, such as cancer cell proliferation, apoptosis, and metastasis. ${ }^{6}$ The dysregulation of miRNAs has been validated in the initiation and development of lung cancer. ${ }^{7}$ For example, miRNA-148a is significantly downregulated in primary NSCLC tissues and can inhibit NSCLC cell migration and invasion by targeting $\mathrm{Wnt}^{8}{ }^{8}$ miRNA-744-5p shows a decreased expression in NSCLC cell lines, while overexpression of miRNA-744-5p inhibits NSCLC cell proliferation, colony formation, and cell invasion in vitro by targeting paired box 2. ${ }^{9}$ Moreover, miR-210-3p is known to be involved in multiple human cancers, such as bladder cancer, breast cancer, and gastric carcinoma, via regulation of a series of target genes. ${ }^{10}$ Zhang et al demonstrated that miR-210-3p is a promising biomarker for the treatment of lung cancer. ${ }^{11}$ In addition, microarray analysis in the present study has proven that upstream stimulating factor 1 (USF1) is a target gene of miR$210-3 p$. USF1 is regarded as a common transcription factor in mammalian cells, which is isolated from HeLa cells and encodes a 43-kDa polypeptide. ${ }^{12}$ Also, the effects of USF1 have been demonstrated in lung cancer. ${ }^{13}$ Furthermore, USF1 DNA binding transcription factor interacts with PCGF3 protein, encoded by the gene. $^{14}$ The protein consists of a C3HC4-type RING finger, which is a known motif implicated in proteinprotein interaction, and the expression of RING1 protein might be an independent prognostic indicator for NSCLC, ${ }^{15}$ but it is unclear whether PCGF3 may be involved in tumor progression in patients with lung cancer. Nevertheless, understanding of the pivotal miR210-3p/USF1/PCGF3 axis associated significantly with the progression of lung cancer is limited. Therefore, the present study aimed to explore the role of the discovered miR-210-3p/USF1/PCGF3 axis in the pathogenesis of lung adenocarcinoma and the underlying mechanisms, which might provide a novel direction for treating lung adenocarcinoma.

\section{Materials and Methods}

\section{Ethics Statement}

Informed consent was obtained from the subjects, and the protocol was approved by the Ethics Committee of People's Hospital of Ningxia Hui Autonomous Region. The animal studies were approved by the Institutional Animal Care and Use Committee of the hospital. The experimental procedures complied with the Helsinki declaration. All animal experiments were carried out according to the published recommendations in the Guide for the Care and Use of Laboratory Animals with minimum suffering.

\section{Human Tissue Specimen Collection}

A total of 50 pairs of tumor and adjacent tumor lung tissues were surgically resected from lung cancer patients (28 males and 22 females) admitted to our hospital from January 2010 to January 2014. None of the patients had received radiotherapy, chemotherapy, or immunotherapy before surgery. The age of 50 NSCLC patients was 2380 (average, $51.22 \pm 9.50$ ) years. After surgery, all patients were followed up for 8-60 months until January 2019. The overall survival was defined as the time duration from exenteration to the last contact or date of death, and the overall survival probabilities were estimated using the Kaplan-Meier method.

\section{Cell Lines}

We purchased four types of human lung cancer cell lines, A549 (3111C0001CCC000002), A-427 (3111C0001CCC000213), NCI-H209 (3111C0001CCC000045), and NCI-H23 (3111C0001CCC000255), two types of human normal lung epithelial cell line BEAS-2B (3131C0001000200027), and embryonic lung fibroblast cell MRC-5 (3111C0001CCC000044) from the Institute of Basic Medical Sciences, Chinese Academy of Medical Sciences. A549, A-427, NCI-H23, and MRC-5 cells were cultured in the medium containing $8 \%$ DMSO and $20 \%$ fetal bovine serum (FBS), NCI-H209 in 20\% FBS/RPMI1640 (w/o HEPES), and BEAS2B cells were cultured in 10\% FBS/DMEM (high glucose). The cells were cultured in the incubator $\left(37^{\circ} \mathrm{C}, 5 \% \mathrm{CO}_{2}\right)$ (Jinan Beisheng Medical Devices Inc., Shandong, China).

\section{RNA Interference}

Based on the oligonucleotides of USF1 and PCGF3 genes in the GenBank of the National Center for Biotechnology Information (NCBI) database, USF1- and PCGF3-specific shRNA were designed. Subsequently, we identified two shRNAs for USF1 (named sh-USF1-1 and sh-USF1-2) and two for PCGF3 (named sh-PCGF3-1 and sh-PCGF3 -2) using BLAST. These shRNAs were constructed into pGPU6/Neo vectors (C02003, GenePharma, China) and transformed into MRC-5 cells, with scrambled shRNA as the negative control, respectively. The sequences are listed in Table 1. 


\section{Luciferase Assays}

Oligonucleotides on the 3'UTR of USF1 mRNA containing the putative miR-210-3p binding sites (USF1-Wt) and oligonucleotides on the $3^{\prime} \mathrm{UTR}$ of $P C G F 3$ mRNA containing the putative USF1 binding sites (PCGF3-Wt) were inserted into the pMIR-reporter vectors (Beijing Huayueyang Biotechnology Co., Ltd, Beijing, China) on SpeI and HindIII sites, respectively. Next, we mutated the putative miR-210-3p binding sites on the $3^{\prime} \mathrm{UTR}$ of USF1 mRNA (USF1-Mut) and the putative USF1 binding sites on the 3'UTR of PCGF3 mRNA (named PCGF3-Mut) and inserted these mutant oligonucleotides into the pMIRreporter vectors, respectively. The reporter vectors containing USF1-Wt or USF1-Mut with miR-210-3p mimic were transfected into HEK-293T cells; also, the reporter vectors containing PCGF3-Wt or PCGF3-Mut plus USF1 were transfected into HEK-293T cells. The overexpression vector of USF1 (named oe-USF1) connects the CDS region to the vector pcDNA3.1. The luminescence of firefly luciferase was determined using dual-luciferase reporter assay system kit (K801-200, BioVision, Milpitas, CA, USA) and Glomax 20/20 luminometer (Promega, Madison, WI, USA) as per the instructions provided by the manufacturer and normalized to that of renilla luciferase. All vectors and miR-210-3p mimic were purchased from GenePharma. The primers of clonal and mutant PCR are listed in Supplementary Table 1.

\section{RNA Immunoprecipitation (RIP) Assays}

A correlation was evaluated between USF1 and AGO2 using a commercial kit (Millipore, Billerica, MA, USA) in accordance with the manufacturer's instructions. Briefly, A549 cells were lysed, and extracts were obtained by centrifugation $\left(14,000 \times g, 4^{\circ} \mathrm{C}, 10 \mathrm{~min}\right)$. The cell extracts were immunoprecipitated with protein A/G Sepharose beads conjugated with either anti-AGO2 antibody (ab32381, 1:50, Abcam, UK) or normal mouse IgG (ab109489, 1:100, Abcam). After incubation with proteinase K (New England Biolabs, Beverly, MA, USA), immunoprecipitated RNA and total RNA and input controls were extracted for real-time quantitative polymerase chain reaction (qPCR) analysis using $U S F 1$-specific primer sequences.

\section{Chromatin Immunoprecipitation (ChIP) Assays}

The enrichment of USF1 in the PCGF3 promoter region was evaluated by ChIP assay following the instructions provided by the kit's manufacturer (Millipore). Briefly, A549 cells were fixed with $10 \%$ formaldehyde for 10 min to generate DNA-protein cross-links. The cell lysates were sonicated to generate chromatin fragments, following which, chromatin fragments were split into three parts: two were immunoprecipitated with either anti-USF1 antibody (ab125020, 1:10,000, Abcam) or normal mouse IgG (ab172730, 1:1000, Abcam) at $4{ }^{\circ} \mathrm{C}$ overnight. The third part is used for quality inspection of DNA lysis. The DNA-protein complexes were precipitated using Protein Agarose/Sepharose (BioVision, Milpitas, CA, USA). The immunoprecipitate was de-crosslinked, and the DNA samples were extracted by a standard phenol/chloroform procedure and analyzed by real-time qPCR analysis using $P C G F 3$-specific primer sequences (forward: 5' AAGGCTATTTCCCACTCCC-3'， reverse: 5'-CGCTCG CTTCTGAATACCTC-3').

\section{Real-Time qPCR}

Total RNA was extracted from A549 cells in mouse tumor xenografts were obtained using TRIzol reagents (15596026, Invitrogen, Carlsbad, CA, USA) and reverse transcribed into cDNA using a commercially available kit (RR047A, TaKaRa, Tokyo, Japan) in accordance with the manufacturer's protocol. Real-time qPCR was performed using TaqMan MicroRNA Assay and TaqMan ${ }^{\circledR}$ Universal PCR Master Mix to quantify the expression of miR-2103p, against $U 6 \mathrm{mRNA}$ expression for normalization. Then, real-time qPCR was performed using the SYBR Premix EX Taq kit (RR420A, TaKaRa) to quantify the expression of ASCL1, SIN3A, USF1, STAT1, and YY1, against $\beta$-actin mRNA expression for normalization. Real-time qPCR was carried out on the ABI PRISM ${ }^{\circledR} 7300$ instrument (Applied Biosystems, USA). Data were calculated by using $2^{-\Delta \Delta C T}$ method. Primers (Table 2) were synthesized by Sangon (Shanghai, China).

Table I Oligonucleotides of Two Pieces of USFI-Specific shRNA, Two Pieces of PCGF3-Specific shRNA and Scrambled shRNA

\begin{tabular}{|l|l|}
\hline shRNA & Oligonucleotides (5'-3') \\
\hline sh-USFI-I & 5'-CCCTTGATCTCAGCTGGATTCTCTT-3' \\
sh-USF2-2 & 5'-CGGCTTCTTTCCGGCTTCACAAATA-3' \\
sh-PCGF3-I & 5'-ACCGAGTGCTTACACACCTTCTGTA-3' \\
sh-PCGF3-2 & 5'-CACCTTCTGTAGAAGCTGCCTTGTA-3' \\
Scrambled shRNA & 5'-TTCTCCGAACGTGTCACGTTT-3' \\
\hline
\end{tabular}




\section{Immunoblotting}

Proteins were extracted from A549 cells in mouse tumor xenografts. An equivalent of $30 \mu \mathrm{g}$ protein samples was separated on $10 \%$ sodium dodecyl sulfate polyacrylamide gel electrophoresis (SDS-PAGE) (AP15L534, LIFE iLAB BIO Technology CO., LTD. Shanghai, China) (80 V/35 $\min , 120 \mathrm{~V} / 45 \mathrm{~min}$ ) and transferred to the polyvinylidene fluoride (PVDF) membrane (Millipore, Billerica, MA, USA). The membranes were probed with rabbit antiUSF1 antibody (ab125020, 1:10,000, Abcam), antiPCGF3 antibody (ab210804, 1:500, Abcam), anti-MMP -2 antibody (ab125020, 1:1000, Abcam), anti-MMP-9 antibody (ab73734, 1:1000, Abcam), anti-Bcl-2 antibody (ab182858, 1:2000, Abcam), anti-Bax antibody (ab32503, 1:2000, Abcam), and anti-GAPDH antibody (ab181602, $1: 10,000$, Abcam), and then incubated with the corresponding horseradish peroxidase-labeled $\mathrm{IgG}$ antibody (ab6721, 1:10,000, Abcam). GAPDH was used as a loading control for normalization. The immunoreactive bands were visualized using the enhanced chemiluminescence (ECL) method (BB-3501, Amersham, UK) according to the manufacturer's protocol. Image Pro Plus software (version 6.0, Media Cybernetics Inc., Rockville, MD, USA) was used to analyze the gray value of each band against GAPDH as the internal reference for relative quantitative analysis of the target protein.

\section{CCK-8 Assays}

A549 cells were cultured for $0,1,2,3$, and 4 days. Then, $10 \mu \mathrm{L}$ CCK-8 reagent was added to each well and incubated for an additional $4 \mathrm{~h}$. The absorbance (at $450 \mathrm{~nm}$ ) was measured on a Microplate reader (BioTek, Vermont, USA), and growth curves plotted.

\section{Flow Cytometric Analysis}

A549 cells $\left(1 \times 10^{6} / \mathrm{mL}\right)$ were stained with fluorescein isothiocyanate (FITC)-Annexin V/propidium iodide (PI) (Shanghai Shuojia Biotechnology, China) for 15-30 min in the dark. Flow cytometer (XL, Coulter, USA) was used to detect apoptosis at the emission wavelength of $530 \mathrm{~nm}$ (FITC-Annexin V) and >575 nm (PI).

\section{Transwell Migration and Invasion Assays}

The transwell chamber system (3413, Beijing Youni Biotechnology, China) was used for cell migration and invasion assays. For cell migration assays, A549 cells $\left(1 \times 10^{6}\right.$ cells $\left./ \mathrm{mL}\right)$ in $100 \mu \mathrm{L}$ serum-free DMEM were added into the upper chambers. For cell invasion assays, A549 cells were added into the upper chambers coated with $50 \quad \mu \mathrm{L}$ Matrigel (40111ES08, YeaSen Biotechnology, Shanghai, China) pre-diluted (1:1) with serum-free DMEM. After incubation for $24 \mathrm{~h}$, the cells that migrated to the lower chamber containing $500 \mu \mathrm{L}$ $20 \%$ FBS-supplemented DMEM (for cell migration assays) and $600 \mu \mathrm{L} 20 \%$ FBS-supplemented DMEM (for cell invasion assays) were subject to fixation with $5 \%$ glutaraldehyde and $0.1 \%$ crystal violet staining. The stained cells were counted in five microscopic fields (TE2000, Nikon, China) per well, and the average was calculated.

\section{Mouse Xenograft Experiments}

A total of $18 \mathrm{BALB} / \mathrm{c}$ mice (equal number of males and females), aged 4-5 weeks and weighing 18 to $22 \mathrm{~g}$ (The Laboratory Animal Center of Ningxia Medical University, China), were maintained specific pathogen-free conditions. A549 cells were adjusted into suspensions $\left(5 \times 10^{6}\right.$ cells/ $\mathrm{mL}$ ) using $10 \mathrm{mg} / \mathrm{mL}$ Matrigel (40111ES08, YeaSen Biotechnology, Shanghai, China), and $0.2 \mathrm{~mL}$ of suspensions with $1 \times 10^{6}$ cells were implanted subcutaneously into the scapular region of each mouse. Intravenous injection of miR-210-3p antagomir (GenePharma) alone or with PCGF3-specific shRNA was administered into mice xenografted with A549 cells after implantation duration of 8 days. Briefly, $12.5 \mu \mathrm{g}$ oligonucleotides were diluted into 1 $\mu \mathrm{g} / \mu \mathrm{L}$ and mixed with $12.5 \mu \mathrm{L}$ water and $25 \mu \mathrm{L}$ of $10 \%$ glucose solution $(\mathrm{w} / \mathrm{v})$ to supplement a final volume of 50 $\mu \mathrm{L}$. Simultaneously, Entranster ${ }^{\mathrm{TM}}$ in vivo reagents (1866811-1, Engreen, China) were mixed with $25 \mu \mathrm{L}$ of $10 \%$ glucose solution (w/v) to a final volume of 50 $\mu \mathrm{L}$. A mixture of oligonucleotides and Entranster in vivo reagents was injected into each mouse by the tail vein, rendering a final concentration of $100 \mu \mathrm{g}$ and $50 \mu \mathrm{L}$ Entranster. The growth of lung cancer xenografts in mice was monitored every 3 days after implantation duration of 8 days. At 32 days after implantation, the mice were euthanized by $\mathrm{CO}_{2}$ suffocation, following which tumor xenografts were excised and lymph nodes surrounding the tumor xenografts resected for hematoxylin-eosin (HE) staining.

\section{Statistical Analysis}

Data (mean \pm standard deviation) were representative of three independent experiments (each in triplicate) and 
Table 2 Primer Sequences Used for Real-Time qPCR Analysis

\begin{tabular}{|l|l|}
\hline Target & Primer Sequences (5'-3') \\
\hline miR-2I0-3P & $\begin{array}{l}\text { F: 5'-GTGCAGGGTCCGAGGT-3' } \\
\text { R: 5'-CTGTGCGTGTGACAGCGGCTGA-3' }\end{array}$ \\
\hline ASCLI & $\begin{array}{l}\text { F: 5'-CCCAAGCAAGTCAAGCGACA-3' } \\
\text { R: 5'-AAGCCGCTGAAGTTGAGCC-3' }\end{array}$ \\
\hline SIN3A & $\begin{array}{l}\text { F: 5'-ACCATGCAGTCAGCTACGG-3' } \\
\text { R: 5'-CACCGCTGTTGGGTGATGA-3' }\end{array}$ \\
\hline USFI & $\begin{array}{l}\text { F: 5'-CTGCTGTTGTTACTACCCAGG-3' } \\
\text { R: 5'-TCTGACTTCGGGGAATAAGGG-3' }\end{array}$ \\
\hline STATI & $\begin{array}{l}\text { F: 5'-CAGCTTGACTCAAAATTCCTGGA-3' } \\
\text { R: 5'-TGAAGATTACGCTTGCTTTTCCT-3' }\end{array}$ \\
\hline YYI & $\begin{array}{l}\text { F: 5'-ACGGCTTCGAGGATCAGATTC-3' } \\
\text { R: 5'-TGACCAGCGTTTGTTCAATGT-3' }\end{array}$ \\
\hline$\beta$-actin & $\begin{array}{l}\text { F: 5'-AAGAGCTACGAGCTGCCTGA-3' } \\
\text { R: 5'-GGCAGTGATCTCCTTCTGCA-3' }\end{array}$ \\
\hline U6 & $\begin{array}{l}\text { F: 5'-GGCTGGTAAGGATGAAGG-3' } \\
\text { R: 5'-TGGAAGGAGGTCATACGG-3' }\end{array}$ \\
\hline
\end{tabular}

Abbreviations: $F$, forward; $R$, reverse.

analyzed using SPSS 21.0 software (IBM, Armonk, NY, USA), with two-tailed $p<0.05$ as a level of statistical significance. For statistical comparison, paired or unpaired $t$-test, a one-way analysis of variance (ANOVA) with Tukey's test, and repeated measurements ANOVA with Bonferroni test were performed.

\section{Results}

\section{Upregulated miR-210-3p Was Associated with a Poor Prognosis of Lung Cancer}

To identify the expression pattern of miR-210-3p in lung cancer, we quantified the expression of miR-210-3p in 50 tumor lung tissues and matched tumor-free lung tissues by real-time qPCR. The results showed that the expression of miR-210-3p was elevated in tumor lung tissues compared to matched tumor-free lung tissues (Figure 1A). Similarly, we compared the expression of miR-210-3p between lung cancer cell lines (A549, A-427, NCI-H209, and NCI-H23) and normal embryonic lung fibroblast cell line HBE using real-time qPCR. Interestingly, all lung cancer cells exhibited a higher expression of miR-210-3p compared to MRC-5 (Figure 1B). Also, A549 cells showed a maximal fold-change in miR-210-3p expression. Next, to study the correlation between miR-210-3p expression and lung cancer patient survival, the overall survival probabilities of 50 lung cancer patients were estimated using the KaplanMeier method with respect to miR-210-3p expression. The survival data showed that patients with high expression of miR-210-3p had a poor overall survival compared to those with low expression (Figure 1C).

Subsequently, the clinical data of 50 patients with NSCLC enrolled in this study were analyzed, and the correlation between the clinical parameters of these patients and the expression of miR-210-3p was analyzed. We found that the expression of miR-210-3p was not directly related to the patient's age, gender, pathological types, smoking and metastasis. Compared to patients at stage I-II, the expression of hsa-miR-210-3p was enhanced markedly in patients at stage III. The expression of miR-210-3p in the tissues of patients with moderate and high differentiation was significantly higher than that in the tissues of patients with low differentiation (Table 3). Taken together, these data supported the conclusion that miR-210-3p is expressed at a high level in NSCLC.

\section{Inhibition of miR-210-3p Inhibited A549 Cell Viability, Migration, and Invasion While Inducing Apoptosis}

Next, we characterized the functional role of miR-210-3p in lung cancer. A549 cells were treated with miR-210-3pspecific inhibitor (Figure 2A) and assayed for the viability, apoptosis, migration, and invasion in vitro by CCK- 8 test, flow cytometry, and transwell chamber assays. The results displayed inhibition of miR-210-3p by specific inhibitor, which hindered lung cancer cell viability, migration, and invasion but facilitated apoptosis (Figure 2B-D). Subsequently, the expression of pro-apoptotic protein Bax, anti-apoptotic protein Bcl-2, and metastatic markers MMP-2 and MMP-9 were determined by immunoblotting. The data showed that the inhibition of miR-210-3p diminished the expression of Bcl-2, MMP-2, and MMP-9 but increased the expression of Bax (Figure 2E). Bcl-2 and Bax are the most important inhibitors of apoptosis and pro-apoptotic genes. The expression of MMP2 and MMP9 was related to tumor invasion and metastasis. Therefore, this phenomenon illustrated the carcinogenic function of miR-210-3p.

\section{miR-210-3p Targeted the Transcription Factor USFI in A549 Cells}

In the following experiments, we recapitulated the molecular mechanisms underlying miR-210-3p in lung cancer. 
hTFtarget is a comprehensive database to identify the regulatory factors of human transcription and their targets related to lung tissues. Additionally, we searched for predicted miR-210-3p targets in the TargetScan database. Subsequently, five common transcription factors were identified as putative miR-210-3p targets: ASCL1, SIN3A, USF1, STAT1, and YY1 (Figure 3A). Real-time qPCR displayed that ASCL1, SIN3A, USF1, STAT1, and YY1 were poorly expressed in tumor lung tissues compared to matched tumor-free lung tissues (Figure 3B). We also observed that USF1 showed a maximal fold-change between tumor lung tissues and tumor-free lung tissues. The Cancer Genome Atlas (TCGA) and GTEx databases show that USF1 was poorly expressed in lung adenocarcinoma and lung squamous cell carcinoma compared to normal tissues (Figure 3C). The expression of miR-210$3 p$ was negatively correlated with the expression of USF1 in lung cancer tissues (Figure 3D). Immunoblotting analysis also demonstrated the downregulation of USF1 in tumor lung tissues compared to matched tumor-free lung tissues and in lung cancer cell lines (A549, A-427, NCIH209, and NCI-H23) compared to MRC-5 (Figure 3E and F). The regulation of miR-210-3p on USF1 was further demonstrated by luciferase assays; decreased luciferase activity was observed at the promoter of the reporter gene containing USF1-Wt compared to that containing USF1-Mut in the presence of miR-210-3p mimic (Figure $3 \mathrm{G})$. Furthermore, USF1 was enriched at the AGO2 promoter region, as assessed by immunoprecipitation using anti-AGO2 antibody relative to normal IgG (Figure $3 \mathrm{H}$ ), suggesting that USF1 could bind to miR-210-3p.
Similarly, immunoblotting showed an elevated expression of USF1 in response to miR-210-3p inhibition in A549 cells (Figure 3I).

\section{miR-2I0-3p Diminished PCGF3 by Targeting USFI in A549 Cells}

Herein, we attempted to identify genes downstream of USF1. The hTFtarget database showed that USF1 regulates PCGF3 gene. TCGA and GTEx databases showed that PCGF3 was poorly expressed like USF1 in lung adenocarcinoma and lung squamous cell carcinoma compared to normal tissues (Figure 4A). Therefore, we proposed a hypothesis that miR-210-3p diminishes the activity of PCGF3 by targeting USF1. Real-time qPCR and immunoblotting analysis demonstrated that PCGF3 was poorly expressed in tumor lung tissues compared to matched tumor-free lung tissues (Figure 4B and C). The expression of PCGF3 was positively correlated with USF1 in lung cancer tissues (Figure 4D). Immunoblotting analysis also demonstrated downregulated PCGF3 in lung cancer cell lines (A549, A-427, NCI-H209, and NCI-H23) compared to that in MRC-5 cells (Figure 4E). The JASPAR database showed the presence of USF1 binding sites at the PCGF3 promoter region (Figure 4F), which was confirmed by luciferase assays, as evidenced by enhanced luciferase activity at the promoter of the reporter gene containing PCGF3-Wt compared to that containing PCGF3-Mut under USF1 overexpression (Figure 4G). Then, A549 cells were subjected to ChIP assay using antiUSF1 or normal mouse IgG. We found that anti-USF1 immunoprecipitated PCGF3 relative to IgG (Figure 4H),

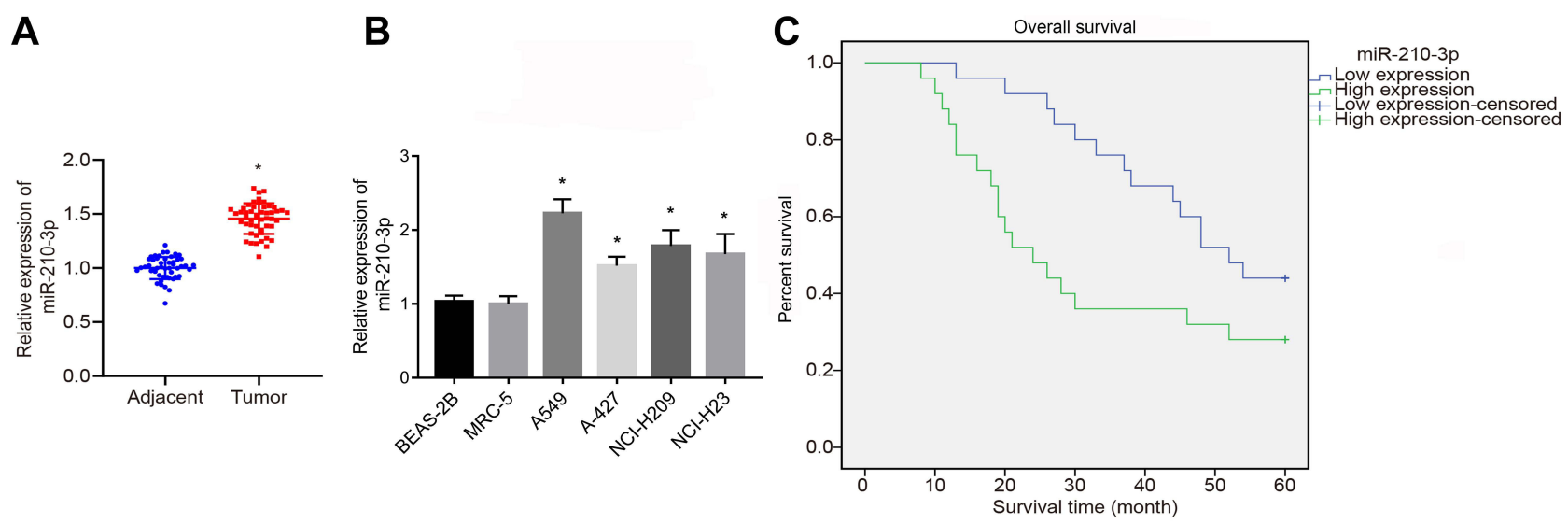

Figure I Upregulated miR-210-3p is associated with poor prognosis of lung cancer. The expression of miR-2I0-3p was analyzed by real-time qPCR in 50 tumor lung tissues compared to matched adjacent tumor tissues $(\mathbf{A})$ and in lung cancer cell lines (A549, A-427, NCl-H209, and NCl-H23) compared to normal lung epithelial cell line BEAS-2B (B). (C) Overall survival probabilities of 50 lung cancer patients were estimated according to miR-2। 0-3p expression using the Kaplan-Meier method and analyzed by Log rank test. ${ }^{*} p<0.05$ compared to matched adjacent tumor tissues by paired $t$-test and to MRC-5 by ANOVA adjusted by Tukey's test. 
Table 3 Correlation Between miR-210-3p Expression and Clinicopathological Parameters of Patients with NSCLC (n, \%)

\begin{tabular}{|c|c|c|c|}
\hline \multirow[t]{2}{*}{ Clinical Features } & \multicolumn{2}{|c|}{ miR-210-3p } & \multirow[b]{2}{*}{$p$-value } \\
\hline & $\begin{array}{l}\text { Low } \\
\text { Expression }\end{array}$ & $\begin{array}{c}\text { High } \\
\text { Expression }\end{array}$ & \\
\hline Gender & & & 0.421 \\
\hline Male & $16(57.14 \%)$ & $12(42.86 \%)$ & \\
\hline Female & 10 (45.45\%) & $12(54.55 \%)$ & \\
\hline $\begin{array}{l}\text { Age }(\text { median age }=58 \\
\text { years) }\end{array}$ & & & 0.545 \\
\hline$<58$ & $9(37.50 \%)$ & $15(62.50 \%)$ & \\
\hline$\geq 58$ & $10(38.46 \%)$ & $16(61.54 \%)$ & \\
\hline Smoking & & & 0.061 \\
\hline Non-smoker & $6(46.15 \%)$ & 7 (53.85\%) & \\
\hline Smoker & $12(32.43 \%)$ & $25(67.57 \%)$ & \\
\hline Clinical stage & & & 0.038 \\
\hline I-II & $6(28.57 \%)$ & 15 (71.43\%) & \\
\hline III & $12(41.38 \%)$ & $17(58.62 \%)$ & \\
\hline Metastasis $(\mathrm{pN})$ & & & 0.154 \\
\hline No-NI & I3 (44.83\%) & $16(55.17 \%)$ & \\
\hline N2 & $8(38.10 \%)$ & $13(61.90 \%)$ & \\
\hline Pathological types & & & 0.204 \\
\hline $\begin{array}{l}\text { Squamous cell } \\
\text { carcinoma }\end{array}$ & 7 (31.82\%) & 15 (68.18\%) & \\
\hline Adenocarcinoma & $8(32.00 \%)$ & 17 (68.00\%) & \\
\hline Others & I (33.33\%) & $2(66.67 \%)$ & \\
\hline Organizational rating & & & 0.027 \\
\hline Moderate and high & $14(41.18 \%)$ & $20(58.82 \%)$ & \\
\hline differentiation & & & \\
\hline Low differentiation & $4(25.00 \%)$ & $12(75.00 \%)$ & \\
\hline
\end{tabular}

suggesting that the transcription factor USF1 binds to PCGF3 and promotes its transcription. To further ascertain the functional regulation of USF1 on PCGF3, two species of sh-USF1 were designed and transfected into A549 cells to establish USF1 knockdown A549 cells. Immunoblotting analysis demonstrated diminished USF1 in A549 cells treated by two pieces of sh-USF1, respectively. We selected the sh-USF1-2 that silenced USF1 profoundly (Figure 4I). Subsequently, we established USF1overexpressed A549 cells using the expression vector containing the USF1 gene and USF1 knockdown A549 cells using sh-USF1. Immunoblotting analysis demonstrated an elevated expression of PCGF3 in USF1-overexpressed A549 cells and decreased expression of PCGF3 in USF1 knockdown A549 cells (Figure 4J). Moreover, A549 cells were treated with miR-210-3p inhibitor and sh-USF1 shRNA. Real-time qPCR and immunoblotting analysis (Figure 4K and L) demonstrated that miR-210-3p inhibitor increases the expression of USF1 and PCGF3 in A549 cells. USF1 knockdown did not affect the expression of miR-210-3p but diminished the expression of PCGF3 in A549 cells treated with miR-210-3p inhibitor. These data proved our hypothesis that miR-210-3p diminishes the activity of PCGF3 by targeting USF1 in A549 cells.

\section{miR-210-3p Facilitated A549 Cell Viability, Migration, and Invasion While Inducing Apoptosis by Inhibiting PCGF3}

Owing to the regulation of miR-210-3p on USF1 and PCGF3, we aimed to dissect whether the miR-210-3p/ USF1/PCGF3 axis is implicated in the development of lung cancer. Two pieces of sh-PCGF3 were designed and independently transfected to achieve PCGF3 knockdown in A549 cells. Immunoblotting analysis demonstrated diminished PCGF3 in A549 cells treated by two shPCGF3, respectively (Figure 5A). The sh-PCGF3 that silenced PCGF3 profoundly was selected for the following studies. Next, A549 cells were treated by miR-210-3p inhibitor and sh-PCGF3, wherein miR-210-3p inhibitionmediated downregulation of USF1 and PCGF3, and PCGF3 knockdown was verified by real-time qPCR and immunoblotting analysis (Figure 5B and C). Subsequently, A549 cells treated with both miR-210-3p inhibitor and shPCGF3 were assayed to evaluate their viability, apoptosis, migration, and invasion in vitro. miR-210-3p inhibition interfered with lung cancer cell viability, migration, and invasion and facilitated apoptosis, which was reversed by PCGF3 knockdown (Figure 5D-F). We also observed elevated expression of MMP-2, MMP-9, and Bcl-2 and decreased the expression of Bax in A549 cells treated with miR-210-3p inhibitor and sh-PCGF3 compared to A549 cells treated with miR-210-3p inhibitor alone (Figure 5G). The above results suggested that miR-210$3 p$ facilitates lung cancer cell viability, migration, and invasion while inducing apoptosis by inhibiting PCGF3.

\section{miR-210-3p Delayed the Development and Metastasis of Lung Cancer in vivo by Inhibiting PCGF3}

Finally, we injected miR-210-3p antagomir alone or with sh-PCGF3 into mice xenografted with A549 cells after implantation duration of 8 days. Real-time qPCR and 

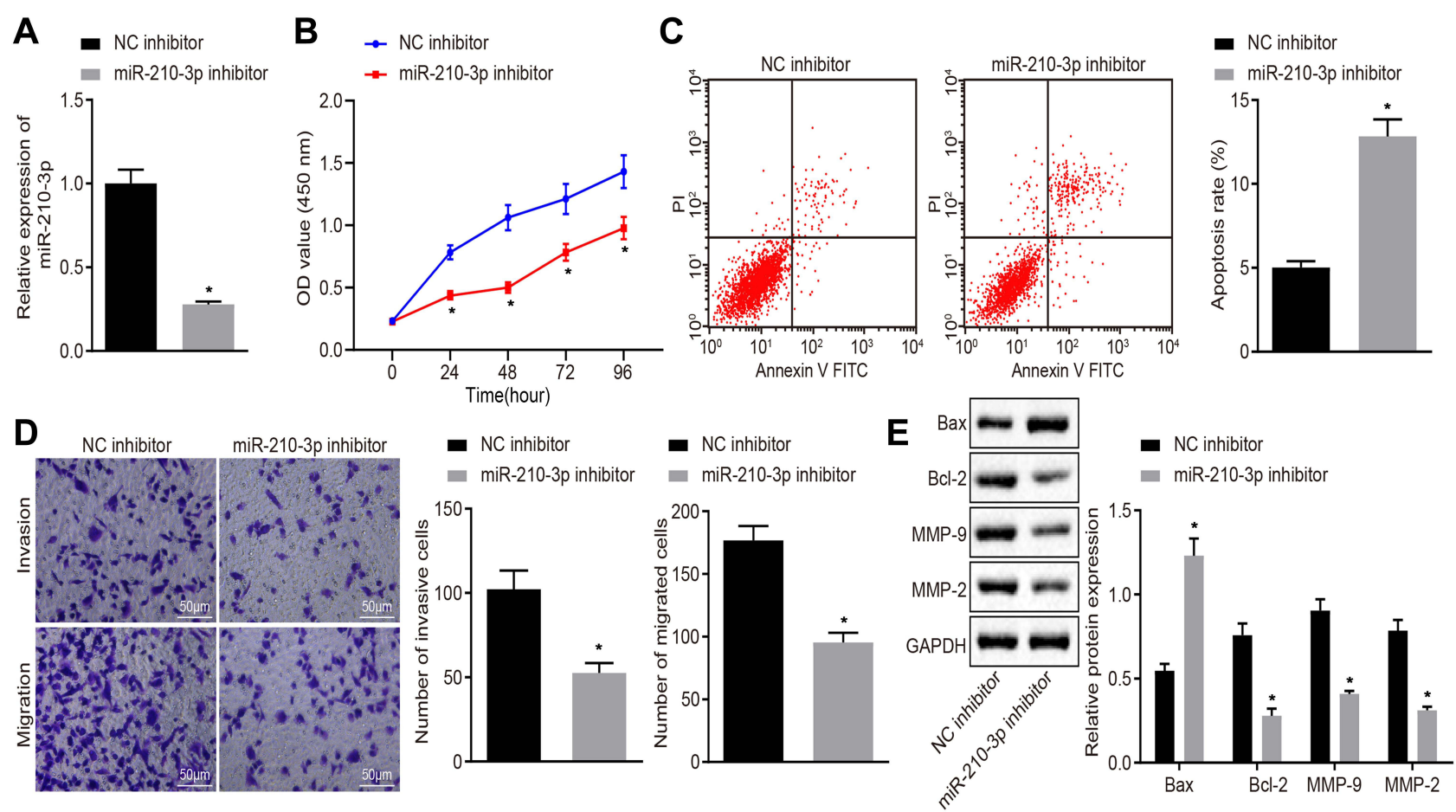

Figure 2 Inhibition of miR-210-3p diminishes A549 cell viability, migration, and invasion while inducing apoptosis. (A) Verification of miR-2I0-3p inhibition in A549 cells treated with miR-210-3p inhibitor by real-time qPCR. (B) Viability of A549 cells treated with miR-210-3p inhibitor was examined by CCK-8 assays. (C) Apoptosis of A549 cells treated with miR-210-3p inhibitor was analyzed by flow cytometry. (D) Representative view $(\times 200)$ of A549 cells treated with miR-210-3p inhibitor migrating from upper transwell chambers into lower well and statistics of migrating cells; Representative view $\times 200)$ of cells invading from Matrigel-coated chambers into lower wells and statistics of invading cells. (E) Immunoblots and quantification of Bax, Bcl-2, MMP-2, and MMP-9 in A549 cells treated with miR-2I 0-3p inhibitor. *p < 0.05 compared to NC inhibitor by unpaired $t$-test or by repeated measurement ANOVA adjusted by Bonferroni test (only for B).

immunoblotting analyzed the miR-210-3p inhibitionmediated downregulation of USF1 and PCGF3 and PCGF3 knockdown in mice (Figure 6A and B). The growth of lung cancer xenografts in mice was monitored every 3 days after implantation duration of 8 days (Figure $6 \mathrm{C})$. The data showed that intravenous injection of miR210-3p antagomir alone delayed the growth of lung cancer xenografts in mice, while intravenous injection of miR$210-3 p$ antagomir and sh-PCGF3 in combination negated the effect of miR-210-3p antagomir on the growth of lung cancer xenografts in mice. Lymph nodes surrounding tumor xenografts were resected for $\mathrm{HE}$ staining. Histopathological changes (Figure 6D) were detected by purplish-red and spindle-shaped cells and fewer lymph nodes in xenograft mice with intravenous injection of miR-210-3p antagomir alone. Compared to these xenograft mice, faded cells and a large number of lymph nodes were observed in xenografted mice with intravenous injection of a combination of miR-210-3p antagomir and sh-PCGF3. Moreover, we performed immunoblotting analysis to determine the expression of Bax, Bcl-2, MMP-2, and MMP-9 in tissues of lung cancer xenografts. The intravenous injection of miR-210-3p antagomir diminished the expression of Bcl-2, MMP-2, and MMP-9 but increased the expression of Bax, which was reversed by intravenous injection of sh-PCGF3 (Figure 6E). These data suggested that miR-210-3p delays the development and metastasis of lung cancer in vivo by inhibiting PCGF3.

\section{Discussion}

Lung cancer is the most frequently occurring aggressive tumor with increasing morbidity worldwide. ${ }^{16}$ The 5-year survival rate of lung cancer is poor due to the high invasion and metastasis of tumors. ${ }^{3}$ In recent years, multiple miRNAs have been reported to play a functional role in the occurrence and progression of lung cancer. ${ }^{5}$ The present study explored the effects of miR-210-3p on cell viability, migration, invasion, and apoptosis in lung cancer. Taken together, the current findings provided evidence that the downregulation of miR-210-3p elevated PCGF3 by upregulating USF1 expression, thereby suppressing the viability, migration, and invasion while inducing apoptosis, which alleviated the progression of lung cancer. 

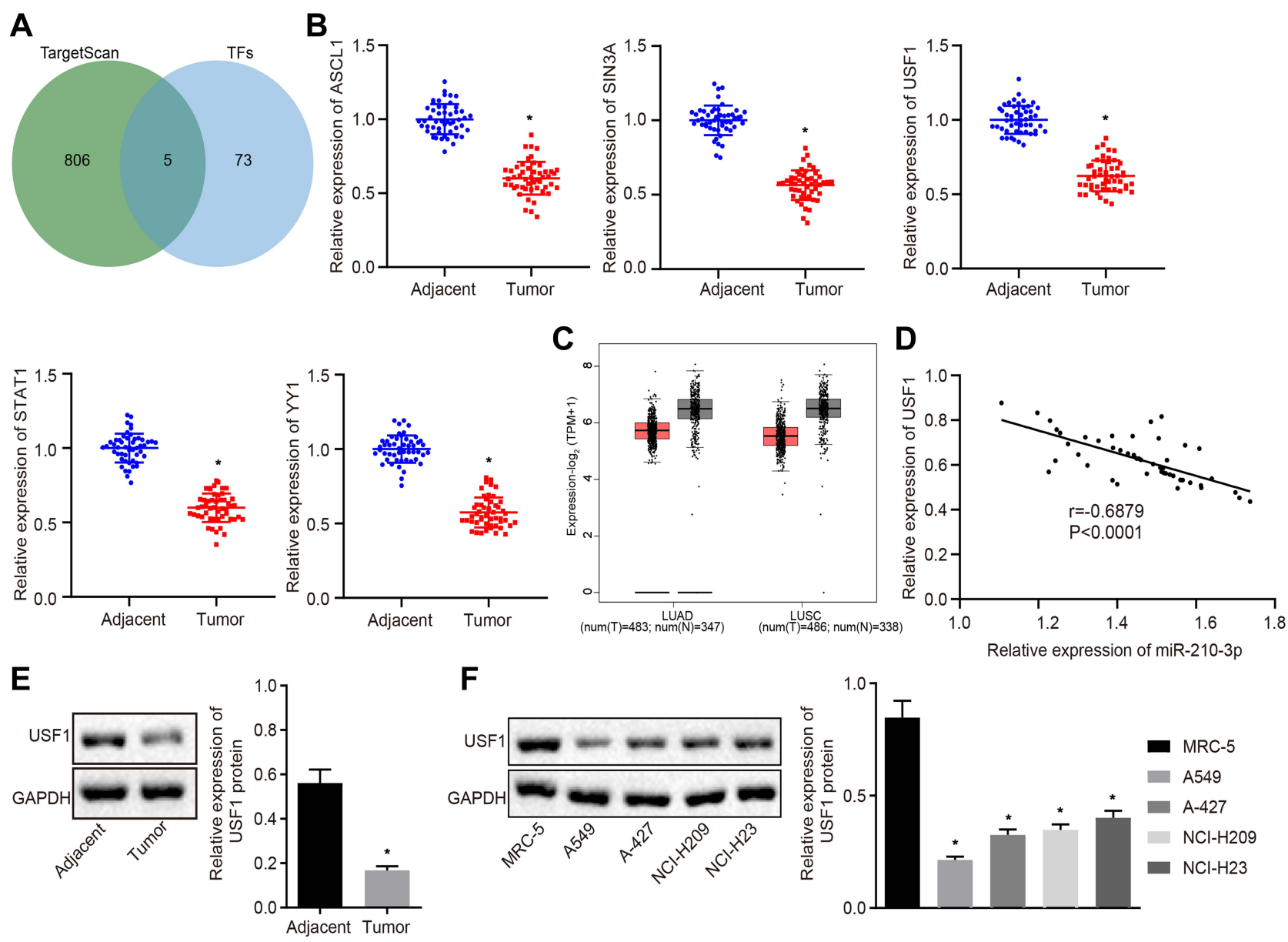

$\mathbf{F}$
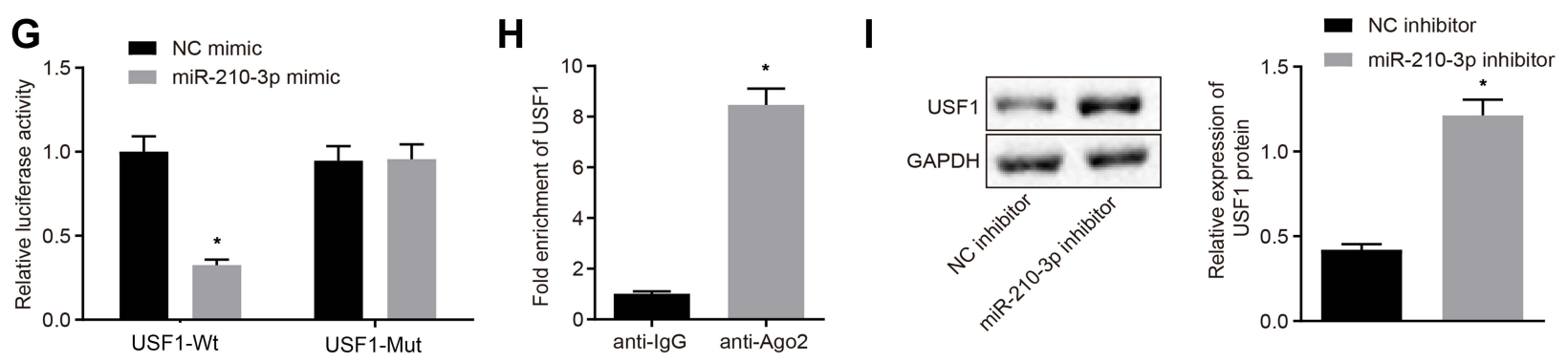

Figure 3 Transcription factor USFI is the target gene of miR-210-3p in A549 cells. (A) Venn plot showing 5 common transcription factors as putative miR-2I0-3p targets, ASCLI, SIN3A, USFI, STATI, and YYI between the hTFtarget database (http://bioinfo.life.hust.edu.cn/hTFtarget\#!/) and TargetScan database (http://www.targetscan.org/ vert_7I/). (B) Real-time qPCR examined the expression of ASCLI, SIN3A, USFI, STATI, and YYI in 50 tumor lung tissues compared to matched tumor-free lung tissues. (C) USFI was poorly expressed in lung adenocarcinoma and lung squamous cell carcinoma compared to normal tissues in TCGA and GTEx databases (http://gepia2.cancer-pku. $\mathrm{cn} / \#$ index). (D) Pearson's correlation analysis demonstrated a negative correlation between the expression of miR-2I0-3p and USFI in lung cancer tissues. (E and $\mathbf{F})$ Immunoblots and quantification of USFI in tumor lung tissues $(n=50)$, matched tumor-free lung tissues $(n=50)$, lung cancer cell lines $(A 549, A-427, \mathrm{NCl}-\mathrm{H} 209$, and $\mathrm{NCl}-$ H23), and MRC-5. (G) Luciferase activity at the promoter of the reporter gene containing USFI-Wt and USFI-Mut in response to miR-2I0-3p-mimic treatment in HEK293 T cells. (H) USFI was enriched at the AGO2 promoter region in the immunoprecipitation using anti-AGO2 antibody relative to normal IgG. (I) Immunoblots and quantification of USFI in A549 cells treated with miR-210-3p inhibitor. ${ }^{*} p<0.05$ compared to matched tumor-free lung tissues by paired $t$-test, to MRC-5 by ANOVA adjusted by Tukey's test, to NC mimic, normal lgG, and NC inhibitor by unpaired $t$-test.

Initially, our results implied that miR-210-3p was upregulated in lung cancer tissues and cells, which was associated with a poor prognosis of the cancer. Thus, it could be speculated that inhibition of miR-210-3p inhibited A549 cell viability, migration, and invasion while inducing apoptosis in lung cancer. Accumulating evidence has recently indicated that aberrant expression of miRNAs is implicated in multiple human cancers, including lung cancer. ${ }^{2}$ For instance, miR-29c regulates cell proliferation and cellular apoptosis that in turn affects the tumor progression of NSCLC. ${ }^{3}$ Existing literature reported that miR$18 \mathrm{a}-5 \mathrm{p}$ is upregulated in NSCLC, and it promotes cell 
A

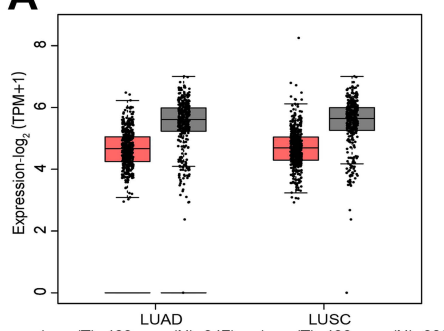

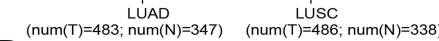

D

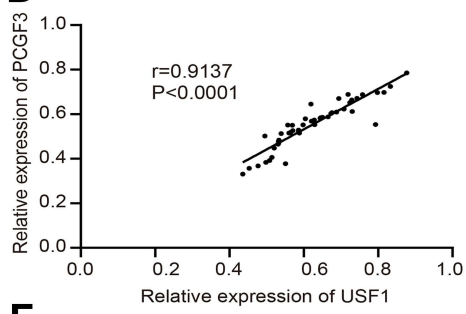

B

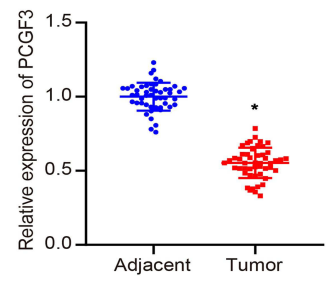

C

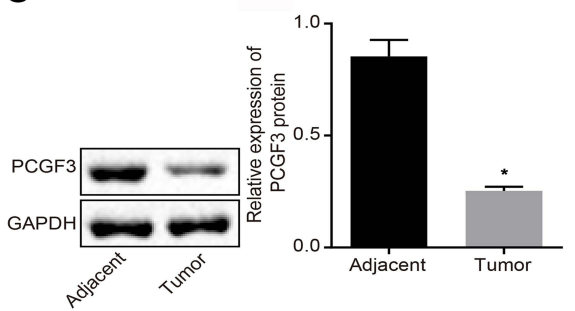

E
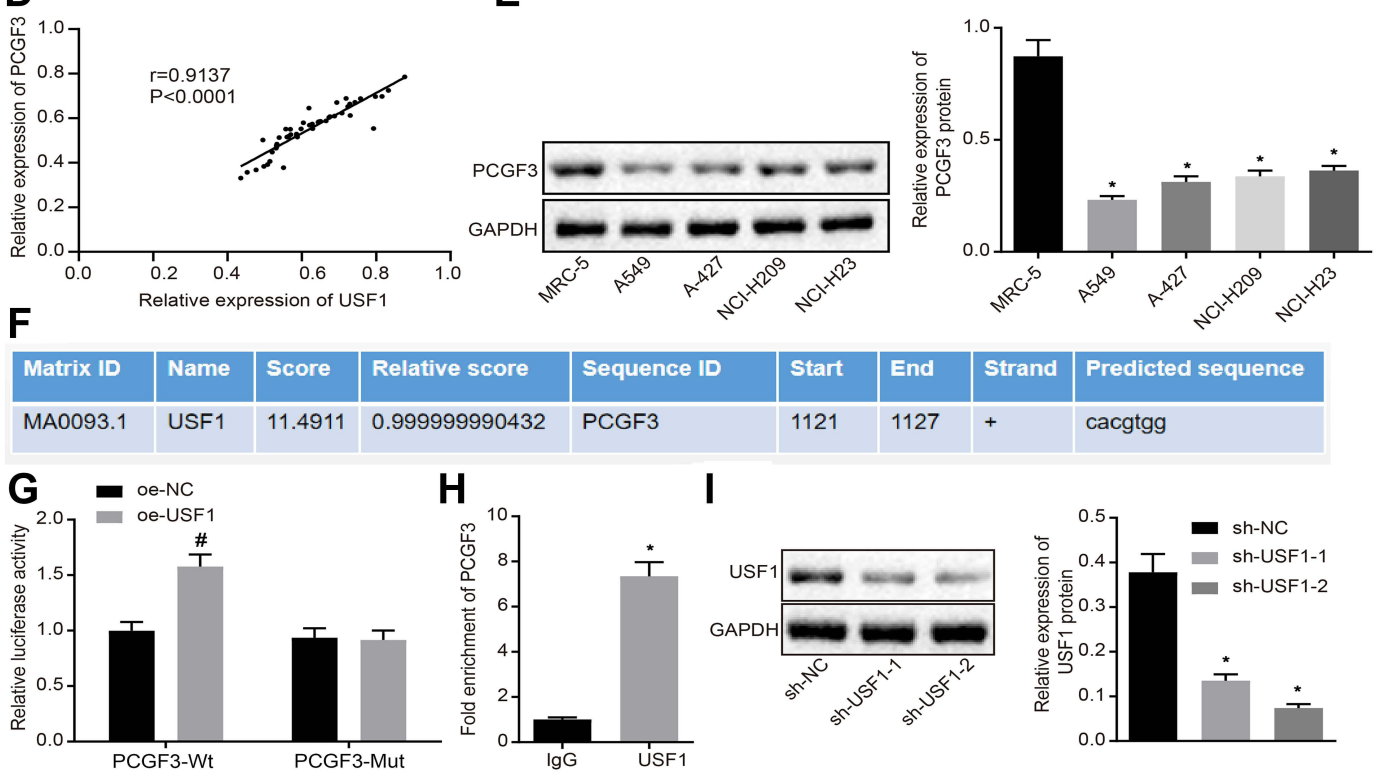

\begin{tabular}{|l|l|l|l|l|l|l|l|l|}
\hline Matrix ID & Name & Score & Relative score & Sequence ID & Start & End & Strand & Predicted sequence \\
\hline MA0093.1 & USF1 & 11.4911 & 0.999999990432 & PCGF3 & 1121 & 1127 & + & cacgtgg \\
\hline
\end{tabular}

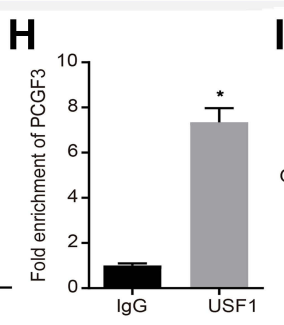

I
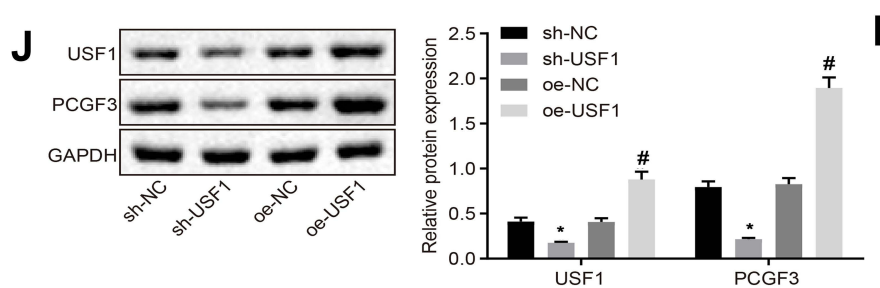

K $\quad{ }^{1.5}=$ NC inhibitor + sh-NC

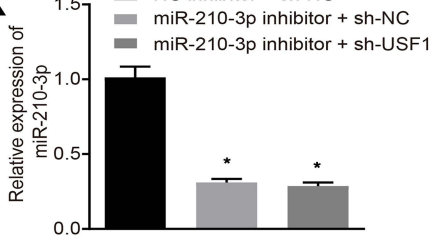

L
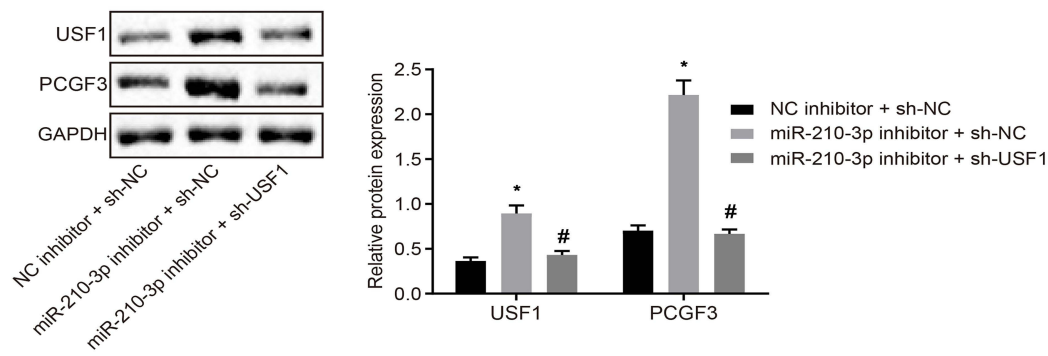

Figure 4 miR-210-3p diminishes the activity of PCGF3 by targeting USFI in A549 cells. (A) PCGF3 was poorly expressed in lung adenocarcinoma and lung squamous cell carcinoma compared to normal tissues in TCGA and GTEx databases. (B) Real-time qPCR examined the expression of PCGF3 in tumor lung tissues ( $\mathrm{n}=50$ ) and matched tumor-free lung tissues $(n=50)$. (C) Immunoblots and quantification of PCGF3 in tumor lung tissues $(n=50)$ and matched tumor-free lung tissues $(n=50)$. (D) Pearson's correlation analysis demonstrated a positive correlation between the expression of PCGF3 and USFI in lung cancer tissues. (E) Immunoblots and quantification of PCGF3 in lung cancer cell lines (A549, A-427, NCl-H209, and $\mathrm{NCl}-\mathrm{H} 23$ ) and MRC-5. (F) Putative USFI binding sites in the PCGF3 promoter region by the JASPAR database analysis. (G) The luciferase activity at the promoter of the reporter gene containing PCGF3-Wt and PCGF3-Mut in response to USFI overexpression treatment in HEK-293T cells. (H) Anti-USFI immunoprecipitated more PCGF3 relative to lgG by ChIP assays. (I) Two sh-USFIs were designed and independently delivered into A549 cells to construct USFI knockdown A549 cells. (J) Immunoblots and quantification of USFI and PCGF3 in A549 cells with USFI overexpression or knockdown. (K) Real-time qPCR examined the expression of miR-210-3p in A549 cells treated with miR-210-3p inhibitor alone or with sh-USFI. (L) Immunoblots and quantification of USFI and PCGF3 in A549 cells treated with miR-210-3p inhibitor alone or sh-USFI. ${ }^{*} p<0.05$ compared to matched adjacent tumor tissues by paired $t$-test, to sh-NC or IgG by unpaired $t$-test, to MRC- 5 or NC inhibitor + sh-NC by ANOVA adjusted by Tukey's test. ${ }^{\#} p<0.05$ compared to oe-NC by unpaired $t$-test and to miR-2I $0-3 p$ inhibitor + sh-NC by ANOVA adjusted by Tukey's test. 
A

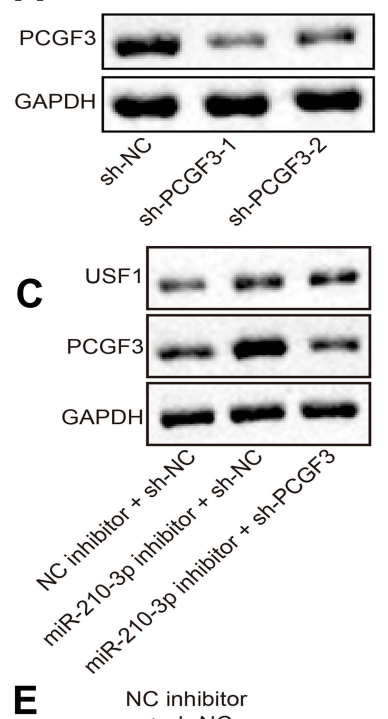

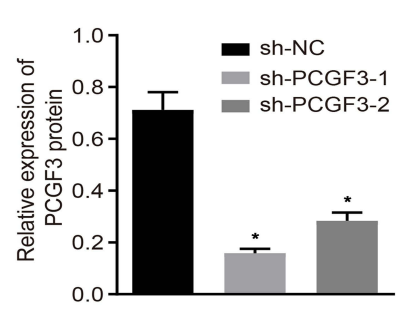

- NC inhibitor + sh-NC

miR-210-3p inhibitor + sh-NC

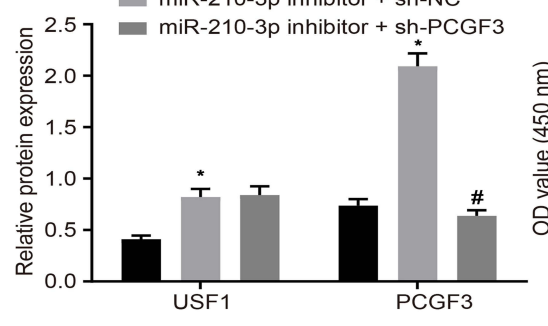

miR-210-3p inhibitor

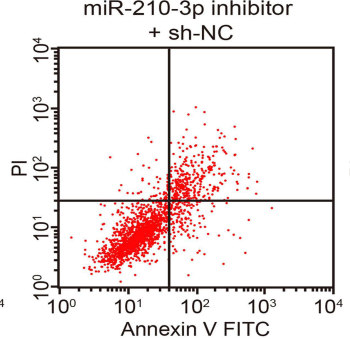

D

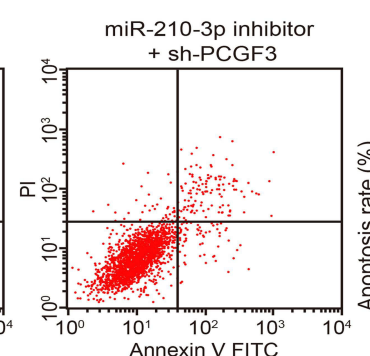

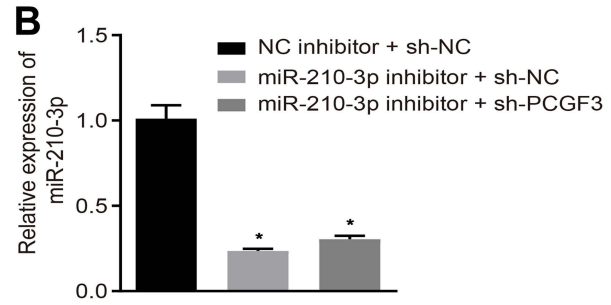

$$
\begin{aligned}
& \rightarrow \quad \text { NC inhibitor + sh-NC } \\
& \rightarrow \text { miR-210-3p inhibitor + sh-NC }
\end{aligned}
$$

$2.07=$ miR-210-3p inhibitor + sh-PCGF3

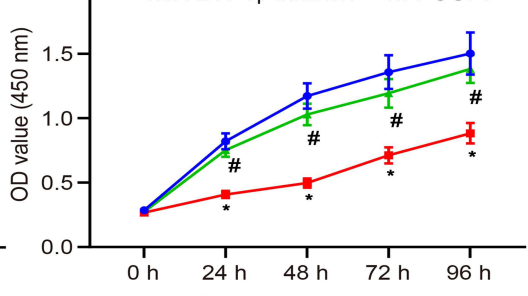

- NC inhibitor + sh-NC

miR-210-3p inhibitor + sh-NC

miR-210-3p inhibitor + sh-PCGF3

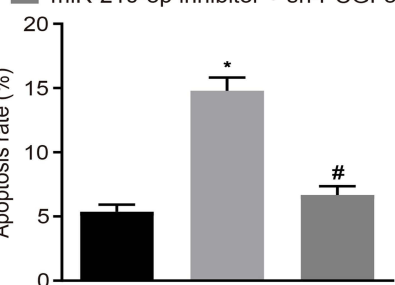

$\mathbf{F}$

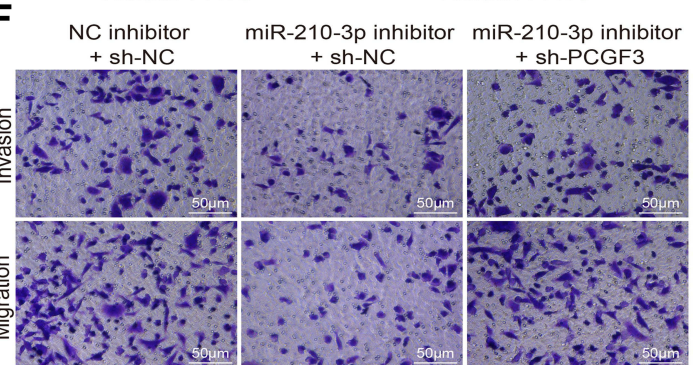

G

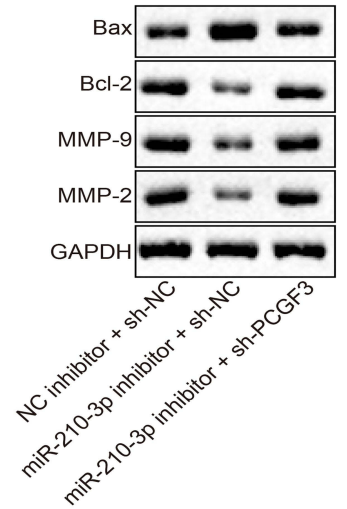

- NC inhibitor + sh-NC miR-210-3p inhibitor + sh-NC

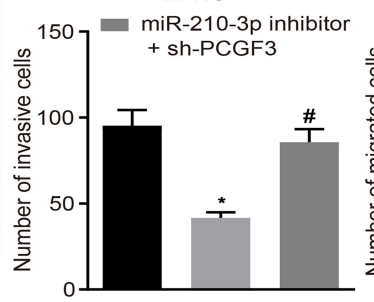

$\mathrm{NC}$ inhibitor + sh-NC miR-210-3p inhibitor + sh-NC

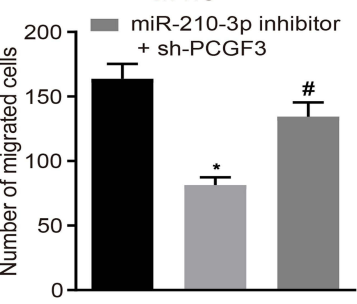

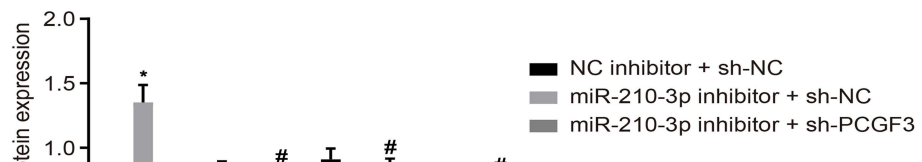

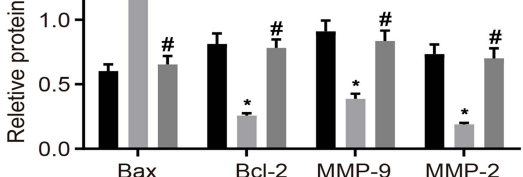

Figure 5 miR-210-3p facilitates A549 cell viability, migration, and invasion but induces apoptosis by inhibiting PCGF3. (A) Two pieces of sh-PCGF3 were designed and independently delivered into A549 cells to construct USFI knockdown A549 cells. (B) Real-time qPCR examined the expression of miR-2I0-3p in A549 cells treated with miR-2I0-3p inhibitor alone or with sh-PCGF3. (C) Immunoblots and quantification of USFI and PCGF3 in A549 cells treated with miR-2I0-3p inhibitor alone or with shPCGF3. (D) Viability of A549 cells treated with miR-210-3p inhibitor alone or with sh-PCGF3 was examined by CCK-8 assays. (E) Apoptosis of A549 cells treated with miR$210-3 p$ inhibitor alone or with sh-PCGF3 was analyzed by flow cytometry. (F) Representative view $(\times 200)$ of A549 cells treated with miR-2I0-3p inhibitor alone or with shPCGF3 migrating from upper into lower chambers and statistics of migrating cells; Representative view $(\times 200)$ of cells invading from Matrigel-coated chambers into lower wells and statistics of invading cells. (G) Immunoblots and quantification of Bax, Bcl-2, MMP-2, and MMP-9 in A549 cells treated with miR-2I0-3p inhibitor alone or with shPCGF3. ${ }^{*} p<0.05$ compared to sh-NC or NC inhibitor + sh-NC and ${ }^{\#} p<0.05$ compared to miR-210-3p inhibitor + sh-NC by ANOVA adjusted by Tukey's test or by repeated measurements ANOVA adjusted by Bonferroni test (only for $\mathbf{D}$ ). 

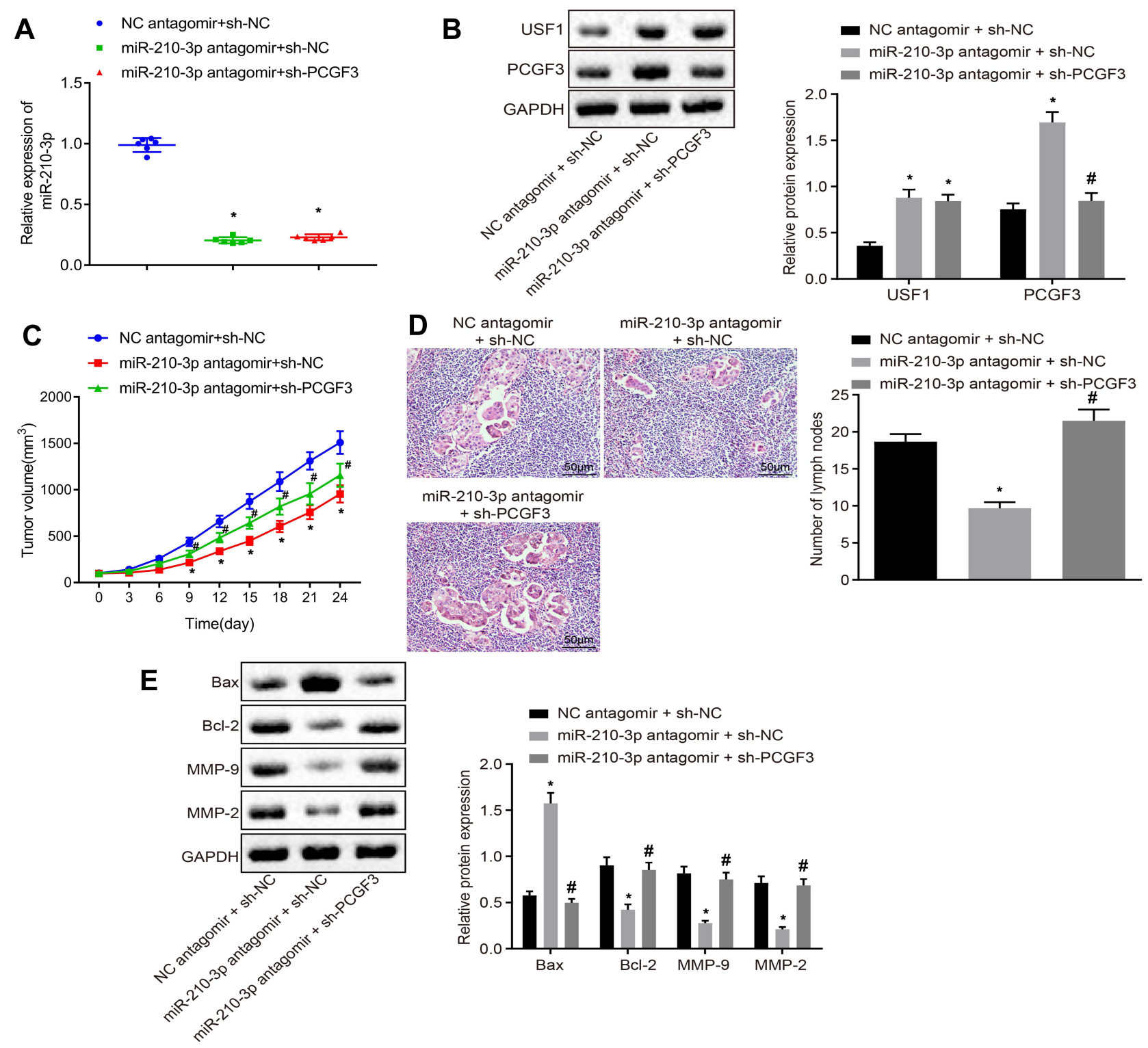

Figure 6 miR-210-3p delays the development and metastasis of lung cancer in vivo by inhibiting PCGF3. (A) Real-time qPCR examined the expression of miR-2/0-3p in tissues of lung cancer xenografts in mice with intravenous injection of miR-210-3p antagomir alone or with sh-PCGF3. (B) Immunoblots and quantification of USFI and PCGF3 in tissues of lung cancer xenografts in mice with intravenous injection of miR-210-3p antagomir alone or with sh-PCGF3. (C) Growth of lung cancer xenografts in mice with intravenous injection of miR-210-3p antagomir alone or with sh-PCGF3 was monitored every 3 days after implantation duration of 8 days. (D) Histopathological changes $(\times 200)$ of lung tissues and lymph node metastasis in xenograft mice with intravenous injection of miR-210-3p antagomir alone or with sh-PCGF3. (E) Immunoblots and quantification of Bax, Bcl-2, MMP-2, and MMP-9 in tissues of lung cancer xenografts in mice with intravenous injection of miR-210-3p antagomir alone or with sh-PCGF3. ${ }^{*} p<0.05$ compared to NC antagomir + sh-NC and ${ }_{p} p<0.05$ compared to miR-210-3p antagomir + sh-NC by ANOVA adjusted by Tukey's test or by repeated measurements ANOVA adjusted by Bonferroni test (only for C). Sample size of six mice in each study group.

proliferation and migration, tumor growth, and metastasis of NSCLC both in vitro and in vivo to aggravate lung cancer. ${ }^{17}$ miR-210 is also shown to play a significant role in a variety of tumor biological functions, including tumor proliferation, survival, and metastasis via regulation of target genes. ${ }^{10}$ Notably, miR-210-3p is highly expressed in different cancers, including lung cancer, which is related to the poor survival rate of lung cancer patients, and it affects the progression of the disease by regulating apoptosis, angiogenesis, and tumor growth. ${ }^{18}$ miR-210-3p also promotes the invasion and EMT of lung cancer cells to enhance the progression of the cancer. ${ }^{11}$ Therefore, silencing of miR-210-3p could be considered as a potential therapeutic target to reverse lung cancer.

Moreover, bioinformatics analysis and dual-luciferase reporter gene assay together validated that $\mathrm{miR}-210-3 \mathrm{p}$ targets 


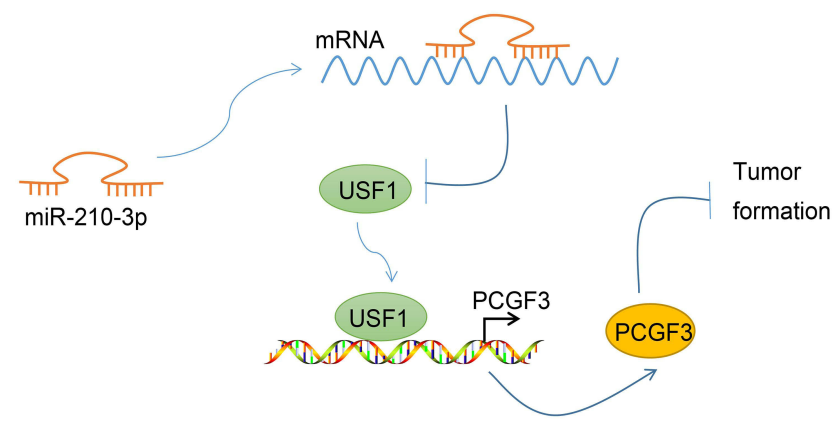

Figure 7 Graphical summary of the mechanism of miR-210-3p in regulating lung cancer progression. miR-210-3p facilitates lung cancer development and metastasis by impairing USFI-mediated promotion of PCGF3.

USF1 and suppresses USF1 expression in lung cancer cells. It is known that miRNAs function as the oncogene or tumor suppressor in the etiology and pathogenesis of cancer by targeting tumor suppressors or oncogenes. ${ }^{6}$ A recent study demonstrated that USF1 acts as a modulator in protein expression and tumor biology. ${ }^{12}$ Furthermore, it has been implicated that USF1, a ubiquitously expressed transcription factor, increases the expression of brain tumors. ${ }^{19}$ Accumulating evidence has also shown that the upregulation of USF1 inhibits lung metastasis that delays the progression of lung cancer, ${ }^{13}$ suggesting that the overexpression of USF1 serves as a biomarker for lung cancer. Taken together, these findings supported that restoration of USF1 underlies the antioncogenic effect of miR-210-3p by stimulating cell viability, migration, and invasion while repressing the apoptosis in lung cancer.

Additionally, the present study confirmed that miR-210-3p diminishes PCGF3 by targeting USF1 in A549 cells. A recent study confirmed that PCGF3 requires an interaction with the USF1 DNA binding transcription factor. ${ }^{14}$ It contains a C3HC4-type RING finger, which is involved in lung cancer via cell cycle and tumor growth regulation ${ }^{15}$; however, the underlying mechanisms of PCGF3 in lung cancer remain to be investigated further. Thus, combining previous studies with our results, a regulatory network could be proposed in the antitumor progression, ie, the depletion of miR-210-3p inhibited A549 cell viability, migration, and invasion while stimulating the apoptosis by promoting USF1 expression to upregulate PCGF3.

\section{Conclusion}

In conclusion, the current study demonstrated that miR-210$3 p$ inhibits the expression of USF1, which potentially suppresses cell viability, migration, and invasion and triggers apoptosis to aggravate lung cancer through inhibition of
PCGF3 expression (Figure 7). This study also provided further insight into the regulatory network and the underlying roles of miR-210-3p-mediated regulation of PCGF3 via USF1 in lung cancer. Nonetheless, A549 is a lung adenocarcinoma cell line, which only represents the type of lung adenocarcinoma in lung cancer, and other types need more matching cell lines for generalization of data. Additional studies are required to adequately define the detailed mechanisms underlying miR-210-3p interaction with USF1 and PCGF3 and the influence on lung cancer progression.

\section{Abbreviations}

miRNAs, microRNAs; NSCLCs, non-small cell lung cancers; 3'-UTR, 3'-untranslated region; USF1, upstream stimulating factor 1; FBS, fetal bovine serum; FITC, fluorescein isothiocyanate; PI, propidium iodide; HE, hematoxylin-eosin; ANOVA, analysis of variance; TCGA, The Cancer Genome Atlas.

\section{Data Sharing Statement}

The datasets used and/or analysed during the current study are available from the corresponding author on reasonable request.

\section{Ethics Approval and Informed Consent}

Subjects were included using informed consent for a protocol approved by the Ethics committee of People's Hospital of Ningxia Hui Autonomous Region [2019] No. 027. Animal studies were performed with the approval from the Institutional Animal Care and Use Committee of People's Hospital of Ningxia Hui Autonomous Region [2019] No. 027.

\section{Funding}

This work was supported by the Ningxia Hui Autonomous Region Key R\&D Program General Project (Fund Code: 
2019BEG03045); Basic research projects of central universities (fund number: 1920160100).

\section{Disclosure}

The authors declare that they have no competing interests.

\section{References}

1. Du X, Zhang J, Wang J, Lin X, Ding F. Role of miRNA in lung cancer-potential biomarkers and therapies. Curr Pharm Des. 2018;23 (39):5997-6010. doi:10.2174/1381612823666170714150118

2. Zhang Y, Wang Y, Wang J. MicroRNA-584 inhibits cell proliferation and invasion in non-small cell lung cancer by directly targeting MTDH. Exp Ther Med. 2018;15(2):2203-2211. doi:10.3892/ etm.2017.5624

3. Zhan S, Wang C, Yin F. MicroRNA-29c inhibits proliferation and promotes apoptosis in non-small cell lung cancer cells by targeting VEGFA. Mol Med Rep. 2018;17(5):6705-6710. doi:10.3892/ mmr.2018.8678

4. Sun F, Li L, Yan P, et al. Causative role of PDLIM2 epigenetic repression in lung cancer and therapeutic resistance. Nat Commun. 2019;10(1):5324. doi:10.1038/s41467-019-13331-x

5. Lu C, Xie Z, Peng Q. MiRNA-107 enhances chemosensitivity to paclitaxel by targeting antiapoptotic factor Bcl-w in non small cell lung cancer. Am J Cancer Res. 2017;7(9):1863-1873.

6. Zhang G, Zheng H, Zhang G, et al. MicroRNA-338-3p suppresses cell proliferation and induces apoptosis of non-small-cell lung cancer by targeting sphingosine kinase 2. Cancer Cell Int. 2017;17:46. doi:10.1186/s12935-017-0415-9

7. Li G, Fang J, Wang Y, Wang H, Sun CC. MiRNA-based therapeutic strategy in lung cancer. Curr Pharm Des. 2018;23(39):6011-6018. doi: $10.2174 / 1381612823666170725141954$

8. Chen Y, Min L, Ren C, et al. miRNA-148a serves as a prognostic factor and suppresses migration and invasion through Wnt1 in non-small cell lung cancer. PLoS One. 2017;12(2):e0171751. doi:10.1371/journal.pone.0171751

9. Chen S, Shi F, Zhang W, Zhou Y, Huang J. miR-744-5p inhibits non-small cell lung cancer proliferation and invasion by directly targeting PAX2. Technol Cancer Res Treat. 2019;18:1533033819876913. doi: $10.1177 / 1533033819876913$
10. Yang X, Shi L, Yi C, Yang Y, Chang L, Song D. MiR-210-3p inhibits the tumor growth and metastasis of bladder cancer via targeting fibroblast growth factor receptor-like 1. Am J Cancer Res. 2017;7 (8):1738-1753.

11. Zhang X, Sai B, Wang F, et al. Hypoxic BMSC-derived exosomal miRNAs promote metastasis of lung cancer cells via STAT3-induced EMT. Mol Cancer. 2019;18(1):40. doi:10.1186/s12943-019-0959-5

12. Ikeda R, Nishizawa Y, Tajitsu Y, et al. Regulation of major vault protein expression by upstream stimulating factor 1 in SW620 human colon cancer cells. Oncol Rep. 2014;31(1):197-201. doi:10.3892/ or.2013.2818

13. Kim KC, Yun J, Son DJ, et al. Suppression of metastasis through inhibition of chitinase 3-like 1 expression by miR-125a-3p-mediated up-regulation of USF1. Theranostics. 2018;8(16):4409-4428. doi: $10.7150 /$ thno. 26467

14. Scelfo A, Fernández-Pérez D, Tamburri S, et al. Functional landscape of PCGF proteins reveals both RING1A/B-Dependent-and RING1A/ B-independent-specific activities. Mol Cell. 2019;74(5):1037-1052. e1037. doi:10.1016/j.molcel.2019.04.002

15. Kitamura S, Tanahashi T, Aoyagi E, et al. Response predictors of S-1, cisplatin, and docetaxel combination chemotherapy for metastatic gastric cancer: microarray analysis of whole human genes. Oncology. 2017;93(2):127-135. doi:10.1159/000464329

16. Wu Y, Zhang J, Hong Y, Wang X. Effects of kanglaite injection on serum miRNA-21 in patients with advanced lung cancer. Med Sci Monit. 2018:24:2901-2906. doi:10.12659/MSM.909719

17. Liang C, Zhang X, Wang HM, et al. MicroRNA-18a-5p functions as an oncogene by directly targeting IRF2 in lung cancer. Cell Death Dis. 2017;8(5):e2764. doi:10.1038/cddis.2017.145

18. Świtlik W, Karbownik MS, Suwalski M, Kozak J, Szemraj J. miR$30 \mathrm{a}-5 \mathrm{p}$ together with miR-210-3p as a promising biomarker for non-small cell lung cancer: a preliminary study. Cancer Biomarkers. 2018;21(2):479-488. doi:10.3233/CBM-170767

19. Zhang L, Handel MV, Schartner JM, et al. Regulation of IL-10 expression by upstream stimulating factor (USF-1) in glioma-associated microglia. $J$ Neuroimmunol. 2007;184(12):188-197. doi:10.1016/j.jneuroim.2006.12.006
OncoTargets and Therapy

\section{Publish your work in this journal}

OncoTargets and Therapy is an international, peer-reviewed, open access journal focusing on the pathological basis of all cancers, potential targets for therapy and treatment protocols employed to improve the management of cancer patients. The journal also focuses on the impact of management programs and new therapeutic agents and protocols on patient perspectives such as quality of life, adherence and satisfaction. The manuscript management system is completely online and includes a very quick and fair peer-review system, which is all easy to use. Visit http://www.dovepress.com/ testimonials.php to read real quotes from published authors. 